\title{
Síndrome da combinação: um relato de prontuário
}

\author{
Síndrome de la combinación: un relato de prontuario \\ Combination syndrome: an accounting report
}

\author{
Adriane MORÁS ${ }^{1}$ \\ César SPIELMANN ${ }^{2}$ \\ Alexandre CONDE ${ }^{3}$ \\ Juliane Pereira BUTZE ${ }^{4}$
}

\begin{abstract}
${ }^{I}$ Graduada do Curso de Odontologia do Centro Universitário da Serra Gaúcha (FSG), 95702-002 Bento Gonçalves-RS, Brasil ${ }^{2}$ Mestre em Prótese Dentária, Professor do Curso de Odontologia do Centro Universitário da Serra Gaúcha (FSG), 95702-002 Bento Gonçalves-RS, Brasil ${ }^{3}$ Doutor em Materiais Dentários, Professor do Curso de Odontologia do Centro Universitário da Serra Gaúcha (FSG), $95702-002$ Bento Gonçalves-RS, Brasil ${ }^{4}$ Doutora em Periodontia, Professora do Curso de Odontologia do Centro Universitário da Serra Gaúcha (FSG), $95702-002$ Bento Gonçalves-RS, Brasil
\end{abstract}

\section{Resumo}

Objetivos: O presente estudo teve como objetivo relatar a análise de um prontuário de paciente portador da Síndrome da Combinação, cuja reabilitação bucal foi realizada através da associação de diferentes tipos de próteses. Materiais e Métodos: Um prontuário de uma paciente que procurou tratamento reabilitador no complexo odontológico da FSG Centro Universitário dentro das disciplinas de Prótese Dentária do curso de Odontologia da FSG Centro Universitário entre os anos de 2017 e 2018 foi escolhido contemplando a condição clínica de necessidade de três modalidades de prótese (prótese total, prótese fixa e prótese parcial removível) ao mesmo tempo para reabilitação, configurando um paciente com Síndrome da Combinação Protética. Resultados: Foi encontrada as seguintes condições 1) Reabsorção óssea na região anterior da maxila; 2) Aumento das tuberosidades; 3) Extrusão dos dentes naturais anteriores mandibulares; 4) Perda óssea abaixo da base de resina acrílica da PPR inferior. Conclusão: Foi possível relatar que, provavelmente, o motivo da instalação da Síndrome da Combinação para o prontuário analisado foi: 1) Ausência de saúde periodontal; 2) Tempo prolongado de uso das próteses antigas; 3) Desarmonia oclusal; e 4) Condição financeira como motivo de retardo na procura do cirurgião-dentista.

Descritores: Síndrome; Prótese Total; Reabilitação Bucal.

\section{Abstract}

Objectives: This study aimed to report the analysis of a patient's chart of the Combination Syndrome, whose oral rehabilitation was performed through the association of different types of prostheses. Materials and Methods: A medical record of a patient who sought rehabilitation treatment at the Complex Dentistry of FSG University Center within the Dental Prosthesis disciplines of the Odontology course at FSG University Center between the years 2017 and 2018 was chosen considering the clinical condition of need for three types of prosthesis (total prosthesis, fixed prosthesis and removable partial prosthesis) at the same time for rehabilitation, configuring a patient with Prosthetic Combination Syndrome. Results: The following conditions were found: 1) Bone resorption in the anterior region of the maxilla; 2) greater tuberosity; 3) Extrusion of anterior mandibular natural teeth; 4) Bone loss below the acrylic resin base of the lower PPR. Conclusion: It was possible to report that, probably, the reason for the installation of the Combination Syndrome for the analyzed medical record was: 1) Absence of periodontal health; 2) Prolonged use of old prostheses; 3) Occlusal disharmony; and 4) Financial condition as a reason for delay in the demand of the dental surgeon.

Descriptors: Syndrome; Denture, Complete; Mouth Rehabilitation.

\section{Resumen}

Objetivos: El presente estudio tuvo como objetivo relatar el análisis de un prontuario de paciente portador del Síndrome de la Combinación, cuya rehabilitación bucal fue realizada a través de la asociación de diferentes tipos de prótesis. Materiales y Métodos: Un prontuario de una paciente que buscó tratamiento rehabilitador en el complejo odontológico de FSG Centro Universitario dentro de las disciplinas de Prótesis Dentales del curso de Odontología de la FSG Centro Universitario entre los años 2017 y 2018 fue elegido contemplando la condición clínica de necesidad de tres modalidades de prótesis (prótesis total, prótesis fija y prótesis parcial removible) al mismo tiempo para rehabilitación, configurando un paciente con Síndrome de la Combinación protética. Resultados: Se han encontrado las siguientes condiciones 1) Reabsorción ósea en la región anterior de la mandíbula; 2) Aumento de las tuberías; 3) Extrusión de los dientes naturales anteriores mandibulares; 4) Pérdida ósea debajo de la base de resina acrílica de la PPR inferior. Conclusión: Fue posible relatar que, probablemente, el motivo de la instalación del Síndrome de Combinación para el prontuario analizado fue: 1) Ausencia de salud periodontal; 2) Tiempo prolongado de uso de las prótesis antiguas; 3) Desarmonía oclusal; y 4) Condición financiera como motivo de retraso en la demanda del cirujano-dentista.

Descriptores: Síndrome; Dentadura Completa; Rehabilitación Bucal.

\section{INTRODUÇÃO}

Nos dias atuais, a maneira dos cirurgiõesdentistas tratarem seus pacientes diante de uma visão multidisciplinar deve ser incorporada permitindo que $\mathrm{o}$ profissional esteja habilitado a entender $\mathrm{e}$ solucionar os casos de forma integral ${ }^{1}$.

Não é incomum a confecção de próteses em pacientes semi-dentados empregando associação de próteses fixas unitárias, parciais removíveis e totais. Esta constatação é referida como Síndrome de Kelly ou Síndrome da Combinação, também caracterizada por apresentar um processo de reabsorção óssea fisiológica da maxila acentuado, principalmente em casos onde há ausência total ou parcial de dentes superiores associados à presença de dentes anteriores inferiores ${ }^{2}$. Também é possível diagnosticar esse quadro por um conjunto de características específicas como o crescimento das tuberosidades, a projeção dos dentes anteriores inferiores, hiperplasia dos tecidos do palato duro e fibromucosa na região anterior e a perda óssea posterior inferior da base da prótese parcial removível ${ }^{3}$. Em pacientes portadores de tal condição, a reabilitação protética é fundamental, pois deve fornecer uma oclusão balanceada para diminuir a pressão excessiva na região anterior da maxila em ambos os contatos cêntricos e excêntricos, através da confecção de novas próteses ${ }^{4}$.

A intervenção do cirurgião-dentista se faz necessária, pois estudos relatam que a ausência de próteses em pacientes totalmente ou parcialmente 
edêntulos, implica diretamente na qualidade de vida, alterando fonação, perda da dimensão vertical da face, estética e função mastigatória ${ }^{5-7}$.

Diante da realidade da Síndrome da Combinação, este trabalho teve como objetivo relatar um caso clínico, através de uma análise de prontuário, de um paciente portador do referido quadro clínico, bem como, relatar, também, a sua reabilitação através da confecção de novas próteses.

\section{MATERIAL E MÉTODO}

A pesquisa iniciou a partir do momento que se obteve aprovação no Comitê de Ética em Pesquisa (CEP) da FSG Centro Universitário (CAAE: 98164218.9.0000.5668). Para tanto, foram analisados os prontuários de pacientes que estiveram em tratamento reabilitador no Complexo Odontológico da FSG Centro Universitário dentro das disciplinas de Prótese Dentária do curso de Odontologia da FSG Centro Universitário entre os anos de 2017 e 2018. Como critérios de inclusão foram adotados: seleção de prontuário que possuíssem o correto e completo preenchimento; presença de registros de imagem do quadro clínico pré e pós-tratamento; e, por último, a conclusão do caso. Os critérios de exclusão foram: prontuários incompletos; prontuários de pacientes que não possuíam associação entre próteses; prontuários que não apresentavam registros fotográficos, ou com precária quantidade de fotografias; e, casos não concluídos. Após esta análise, apenas um prontuário de uma paciente com Síndrome da Combinação foi selecionado.

\section{RESULTADOS}

O prontuário selecionado foi de uma paciente de 60 anos de idade, gênero feminino que se apresentou na clínica do complexo odontológico da FSG Centro Universitário queixando-se de desconforto com as antigas próteses que estavam mal adaptadas devido a problemas periodontais na arcada inferior e pelo prolongado tempo de uso da prótese superior que já havia perdido a função, sendo encontrado o registro de que o motivo da demora da procura pelo cirurgião-dentista fora a falta de condição financeira. $\mathrm{O}$ registro da anamnese detalhada, exame físico e radiográfico, dava conta que a paciente fora diagnosticada com Síndrome da Combinação. A paciente não possuía nenhum dente superior e ausência bilateral de dentes posteriores na arcada inferior (Classe I de Kennedy e modificação 1) perda de dimensão vertical de oclusão e perda de estrutura óssea na base da prótese inferior (Figura 1).

No prontuário foi possível constatar que as condições bucais da paciente foram adequadas através dos seguintes procedimentos: tratamento periodontal e tratamento restaurador. Concluída esta fase, a paciente foi encaminhada para confecção de novas próteses. Primeiramente, foi realizado o reembasamento da prótese superior antiga durante o período em que estava sendo realizada a confecção das novas próteses. Foi constatado no prontuário que o dente 34 estava com a estrutura coronal perdida e foi realizado um tratamento endodôntico, modelagem, fundição e cimentação de núcleo metálico fundido, repreparo, confecção de provisório para coroa metalocerâmica (Figura 2) e, cimentação definitiva da coroa finalizada (Figura 3). Foram confeccionados, também, os preparos dos nichos durante a construção da coroa que serviu como pilar da prótese parcial removível (PPR).

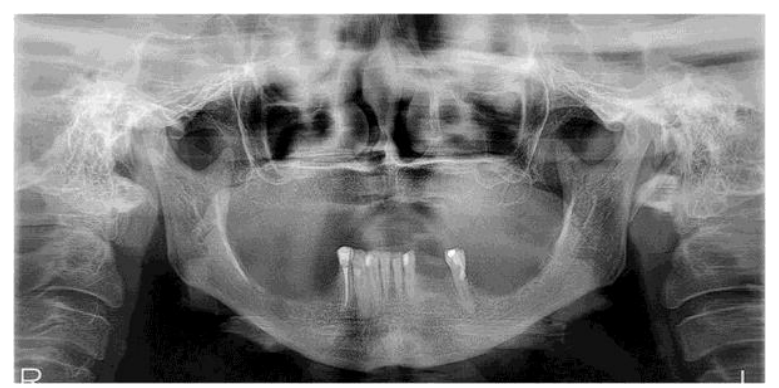

Figura 1: Radiografia panorâmica inicial.

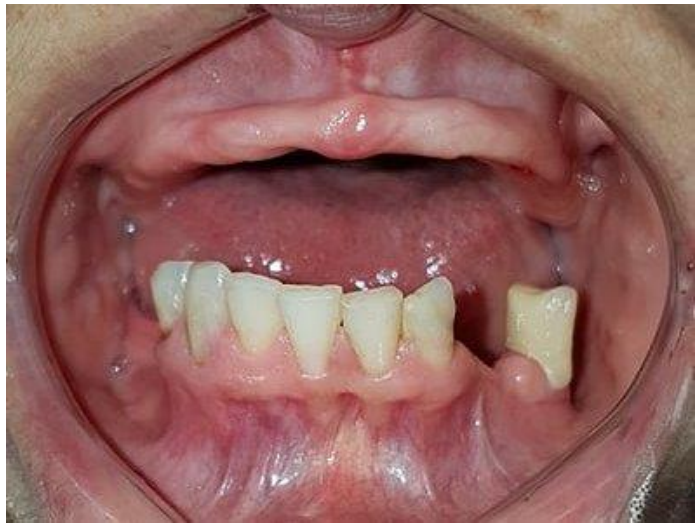

Figura 2: Confecção do dente provisório do elemento 34.

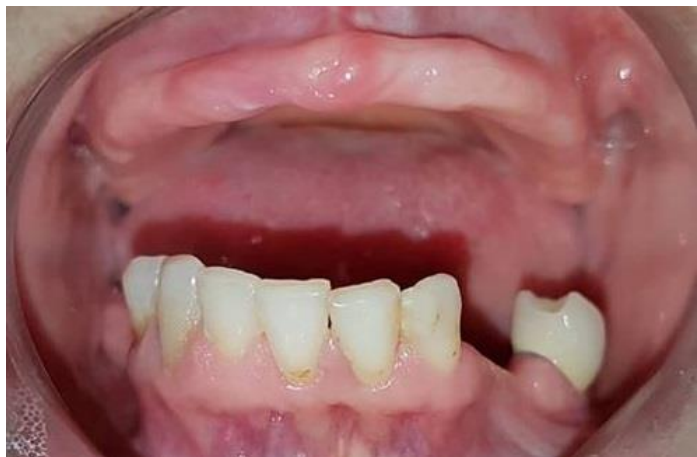

Figura 3: Cimentação definitiva da coroa metalocerâmica do elemento 34.

Foi possível depreender do prontuário que os modelos de estudo foram confeccionados e montados em articulador semi-ajustável que serviram para nortear o plano oclusal da paciente para o planejamento da prótese superior, inferior e planejamento de nichos e grampos. Após moldagem da arcada inferior, foi confeccionada a estrutura metálica em níquel-cromo, enceramento, testes oclusais, funcionais e fonéticos, e em seguida acrilização da PPR inferior. Concomitantemente, foi feita para a arcada superior, moldagem anatômica 
com godiva, modelo de gesso anatômico para confecção de moldeira individual, moldagem funcional com vedamento periférico com godiva de baixa fusão e pasta zinquenólica, modelo funcional, placa base em resina acrílica com rolete de cera, montagem de dentes em cera, testes oclusais, funcionais e fonéticos, acrilização e entrega da prótese total superior (Figuras 4 e 5). Paciente não necessitou de procedimentos cirúrgicos mais invasivos para melhorar sua condição clínica de saúde. A tabela 1 mostra de forma simplificada as constatações clínicas da paciente verificadas em sua análise de prontuário antes e depois do tratamento reabilitador.

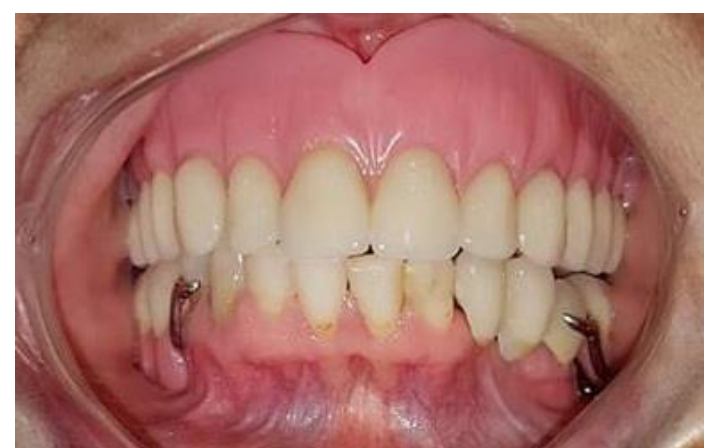

Figura 4: Instalação das próteses total superior, parcial removível inferior e coroa metalocerâmica do elemento 34 .
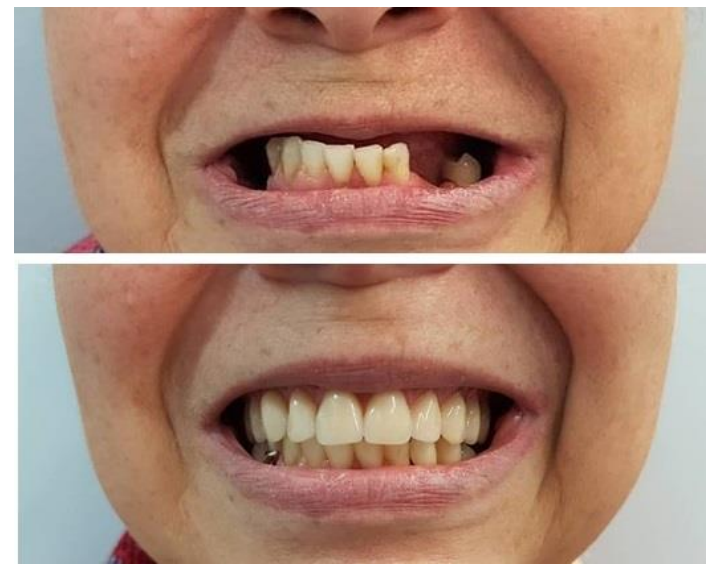

Figura 5: Comparação do antes e após a instalação das próteses definitivas.

Tabela 1: Descrição da condição pré-operatória e pós-operatória. Caxias do Sul, 2018

\begin{tabular}{c|c|c}
\hline Quesito analisado & $\begin{array}{c}\text { Condição } \\
\text { pré-operatória }\end{array}$ & $\begin{array}{c}\text { Condição } \\
\text { pós-operatória }\end{array}$ \\
\hline $\begin{array}{c}\text { Reabsorção óssea na região anterior } \\
\text { da maxila }\end{array}$ & Presente & Presente \\
\hline Aumento das tuberosidades & Presente & Presente \\
\hline Hiperplasia papilar palatina & Ausente & Ausente \\
\hline $\begin{array}{c}\text { Extrusão dos dentes naturais } \\
\text { anteriores mandibulares }\end{array}$ & Presente & $\begin{array}{c}\text { Presente mas com } \\
\text { ajuste oclusal }\end{array}$ \\
\hline $\begin{array}{c}\text { Perda óssea abaixo da base de resina } \\
\text { acrílica da prótese parcial removível } \\
\text { mandibular }\end{array}$ & Presente & Presente \\
\hline Saúde periodontal/gengival & Ausente & Presente \\
\hline Adaptação das próteses & Ausente & Presente \\
\hline Equilíbrio oclusal & Ausente & Presente \\
\hline Dimensão vertical & Perda & Retorno \\
\hline Posicionamento mandibular & Anterior & Ajustado \\
\hline
\end{tabular}

\section{DISCUSSÃO}

O termo Síndrome da Combinação foi proposto em 1972 por $\mathrm{Kelly}^{3}$, tendo como características clínicas reabsorção óssea na região anterior da maxila, aumento das tuberosidades, hiperplasia papilar palatina, extrusão dos dentes naturais anteriores mandibulares e perda óssea abaixo da base de resina acrílica da prótese parcial removível mandibular. Outras alterações adicionais podem favorecer o diagnostico, tais como a perda de dimensão vertical, mandíbula posicionada para anterior e problemas periodontais ${ }^{8}$. Nosso estudo utilizou os parâmetros citados acima como ponto de partida para análise do prontuário.

Ainda não se sabe que fatores desencadeiam as alterações que levam à síndrome da combinação, porém diversos estudos relatam a substituição do osso alveolar por tecido hiperplásico que pode agravar a reabsorção dependendo do tempo de uso prolongado de próteses que geram forças mastigatórias anormais e desarmonia oclusal ${ }^{9,10}$. Em nosso estudo foi possível constatar que a paciente teve a condição financeira como motivo de retardo na procura do cirurgião-dentista, ausência de saúde periodontal, tempo prolongado de uso das próteses antigas e desarmonia oclusal. Possivelmente estes fatores contribuíram no estabelecimento da síndrome da combinação no prontuário da paciente analisada.

Embora existam estudos apontando a necessidade das principais características clínicas estarem presentes no paciente portador da Síndrome da Combinação de forma simultânea, no presente relato não foram observados todos os sinais descritos por Kelly ${ }^{3}$. Em nossa pesquisa foi possível constatar: 1) Reabsorção óssea na região anterior da maxila; 2) Aumento das tuberosidades; 3) Extrusão dos dentes naturais anteriores mandibulares; 4) Perda óssea abaixo da base de resina acrílica da PPR inferior. Não sendo verificada a hiperplasia papilar palatina.

Os achados de nosso trabalho vão ao encontro de outros relatos semelhantes na literatura. Cunha et al. $^{8}$ observaram que, dos pacientes analisados, nenhum apresentou todos os sinais concomitantemente, em contrapartida todos os pacientes apresentaram os sinais de forma isolada ou parcialmente agrupadas. Corroborando este estudo, Costa et al. ${ }^{11}$, em relato de caso, observaram quatro sinais clínicos dos cinco. Apenas a hiperplasia palatina não pode ser efetivamente caracterizada neste último estudo, semelhante a nossa pesquisa que evidenciou a mesma constatação. Em nosso estudo foi possível corroborar Cunha et al. ${ }^{8}$ também para as alterações adicionais que favorecem o diagnostico, tais como a perda de dimensão vertical, mandíbula posicionada para anterior e problemas periodontais.

O presente trabalho abordou um caso em que a paciente foi reabilitada por meio da combinação de prótese total superior, prótese removível inferior classe I de Kennedy e prótese fixa unitária. Apesar de a literatura ser enfática quanto ao pouco uso desta combinação a fim de se prevenir a síndrome ${ }^{8}$, a condição econômica da paciente foi $o$ fator determinante para a escolha deste tratamento. 
Infelizmente, na grande maioria dos casos, os pacientes já apresentam esta combinação, predispondo às alterações relatadas ${ }^{11}$. Em casos de favorabilidade econômica, é possível prevenir o desenvolvimento da Síndrome da Combinação por meio de uma correta moldagem, visitas periódicas ao dentista $^{12}$, reestabelecimento da oclusão de forma que diminua o estresse oclusal da força mastigatória na região anterior da maxila ${ }^{13}$ e, quando possível, colocação de implantes $^{14,15}$.

Deste modo, quando viável pelo paciente, fica evidente a necessidade de se assumir uma postura preventiva quando da reabilitação de pacientes portadores desta condição. Com o advento dos implantes, é possível a prevenção ou tratamento mais duradouro desta síndrome através da colocação dos dispositivos com rosca osteointegráveis mesmos na região posterior da mandíbula, eliminando a extremidade livre, impedindo assim movimentos verticais e laterais que geram reabsorção óssea ${ }^{12}$. Mas, independentemente do tratamento escolhido, o mais importante é que a carga oclusal seja distribuída igualmente entre ambos os arcos a fim de se devolver a estabilidade oclusal na região posterior ${ }^{1}$.

Embora o presente trabalho tenha se empenhado em analisar o melhor prontuário disponível de caso de Síndrome de Combinação, mais estudos se fazem necessários para melhor elucidar tal condição clínica, além da constatação da necessidade de conscientizar a classe odontológica para registrar mais informações em prontuário que possam contribuir com o desvelar deste assunto.

\section{CONCLUSÃO}

Foi possível relatar na presente análise de prontuário de que estiveram presentes: 1) Reabsorção óssea na região anterior da maxila; 2) Aumento das tuberosidades; 3) Extrusão dos dentes naturais anteriores mandibulares; 4) Perda óssea abaixo da base de resina acrílica da PPR inferior.

Também foi possível constatar que, provavelmente, o motivo da instalação da Síndrome da Combinação para o prontuário analisado foi: 1) Ausência de saúde periodontal; 2) Tempo prolongado de uso das próteses antigas; 3) Desarmonia oclusal; e 4) Condição financeira como motivo de retardo na procura do cirurgião-dentista.

\section{REFERÊNCIAS}

1. Mezzomo E, Suzuki RM. Reabilitação oral contemporânea. São Paulo: Santos; 2012.

2. Goyatá FR, Tostes Vl, Rodrigues CRT, Corga RB, Cunha LG. Síndrome da combinaçãoRelato de Caso Clínico. IJD. Recife. 2010;9(3):160-64.

3. Kelly E. Changes caused by a mandibular removable partial denture opposing a maxillary complete denture. J Prosthet Dent. 1972; 27(2):140-50.

4. Nogueira RP, Miraglia SS, Soares FAV. Considerações sobre síndrome da combinação (Kelly) na clínica odontológica reabilitadora. PCL-Rev bras prótese clín lab. 2002;4(19):218-22.

5. Campos MTFS, Monteiro JBR, Ornelas APRC. Fatores que afetam o consumo alimentar e a nutrição do idoso. Rev Nutr. 2000;13(3):157-65.

6. Silva MES, Magalhães CS, Ferreira EF. Perda dentária e expectativa da reposição protética: estudo qualitativo. Ciênc saúde coletiva. 2010; 15(3):813-20

7. Carvalho LF, Melo JRO, Carvalho FAA, Ramos JG, Lima RA. O impacto do edentulismo na qualidade de vida de pacientes edêntulos. Rev ACBO. 2019;8(1):40-8.

8. Cunha LDAP, Rocha EP, Pellizzer EP. Prevalência da Síndrome de Kelly em usuários de prótese parcial removível. RGO. 2007; 55(4):325-28.

9. Palmqvist S, Carlsson GE, Owall B. The combination syndrome: a literature review. J Prosthet Dent. 2003; 90(3):270-75.

10. Crawford RWI, Walmsley AD. A review of prosthodontic management of fibrous ridges. $\mathrm{Br}$ Dent J. 2005;199(11):715-19.

11.Costa MM, Cardoso GM, Ferreira Júnior LH, Resende CD, Tavares LN, Rocha FS et al. Síndrome da Combinação: diagnóstico, prevenção e considerações sobre o tratamento Rev Odontol Bras Central 2016;25(72):59-64.

12. Giampaolo ET. Síndrome da combinação em próteses parciais removíveis: sinais, sintomas e plano de tratamento. In: Vanzillotta PS, Salgado LPS. Odontologia integrada: atualizaçäo multidisciplinar para o clínico e o especialista. Rio de Janeiro, Pedro Primeiro, 2001: Pedro Primeiro; 2001.

13. Saunders TR, Gillis RE Jr, Desjardins RP. The maxillary complete denture opposing the mandibular bilateral distalextension partial denture: treatment considerations. J Prosthet Dent. 1949;41(2):124-28.

14. Hansen CA, Jaarda MJ. Treatment alternatives for modified combination syndrome. Gen Dent. 1990;38(2):132-37

15.Salvador MCG, Valle AL, Ribeiro MCM, Pereira JR. Assessment of the prevalence index on signs of combination syndrome patients treated at Bauru School of Dentistry, University of São Paulo. J Appl Oral Sci. 2007;15(1):9-13. 


\section{CONFLITO DE INTERESSES}

Os autores declaram não haver conflitos de interesse.

\section{AUTOR PARA CORRESPONDENCIA}

\section{Juliane Pereira Butze}

juliane.butze@fsg.edu.br

Submetido em 12/11/2018

Aceito em 27/02/2019 